\title{
Is small fiber neuropathy induced by gadolinium-based contrast agents?
}

Radbruch, Alexander ; Richter, Henning ; Bücker, Patrick ; Berlandi, Johannes ; Schänzer, Anne ; Deike-Hofmann, Katerina ; Kleinschnitz, Christoph ; Schlemmer, Heinz-Peter ; Forsting, Michael ; Paulus, Werner ; Martin, Louise F ; van Thriel, Christoph ; Karst, Uwe ; Jeibmann, Astrid

\begin{abstract}
OBJECTIVES: In recent years, complaints of patients about burning pain in arms and legs after the injection of gadolinium-based contrast agents (GBCAs) have been reported. In the current study, we investigated changes of small fibers in the epidermis as a potential cause of the patient complaints in a mouse model. METHODS: Six groups of 8 mice were intravenously injected with either a macrocyclic GBCA (gadoteridol, gadoterate meglumine, gadobutrol), a linear GBCA (gadodiamide or gadobenate dimeglumine) ( $1 \mathrm{mmol} / \mathrm{kg}$ body weight), or saline ( $\mathrm{NaCl}$ 0.9\%). Four weeks after injection, animals were euthanized, and footpads were assessed using immunofluorescence staining. Intraepidermal nerve fiber density (IENFD) was calculated, and the median number of terminal axonal swellings (TASs) per IENFD was determined. RESULTS: Nonparametric Wilcoxon signed-rank test revealed significantly lower IENFDs for all GBCAs compared with the control group $(\mathrm{P}<0.0001)$ with the linear GBCAs showing significantly lower IENFDs than the macrocyclic GBCAs $(\mathrm{P}<0.0001)$. The linear GBCAs presented significantly more TAS per IENFD than the control group $(\mathrm{P}<0.0001)$, whereas no significant increase of TAS per IENFD compared with the control group was found for macrocyclic GBCAs $(\mathrm{P}<0.237)$. INTERPRETATION: It is unclear whether or at what dosage the decrease of IENFDs and the increase of TAS per IENFD found in the current animal model will appear in humans and if it translates into clinical symptoms. However, given the highly significant findings of the current study, more research in this field is required.
\end{abstract}

DOI: https://doi.org/10.1097/RLI.0000000000000677

Posted at the Zurich Open Repository and Archive, University of Zurich

ZORA URL: https://doi.org/10.5167/uzh-190311

Journal Article

Published Version

Originally published at:

Radbruch, Alexander; Richter, Henning; Bücker, Patrick; Berlandi, Johannes; Schänzer, Anne; Deike-Hofmann, Katerina; Kleinschnitz, Christoph; Schlemmer, Heinz-Peter; Forsting, Michael; Paulus, Werner; Martin, Louise F; van Thriel, Christoph; Karst, Uwe; Jeibmann, Astrid (2020). Is small fiber neuropathy induced by gadoliniumbased contrast agents? Investigative Radiology, 55(8):473-480.

DOI: https://doi.org/10.1097/RLI.0000000000000677 


\title{
Is Small Fiber Neuropathy Induced by Gadolinium-Based Contrast Agents?
}

\author{
Alexander Radbruch, MD, JD, *† Henning Richter, DVM, PhD, f Patrick Bücker, $\S$ Johannes Berlandi, PhD, // \\ Anne Schänzer, MD, 9 Katerina Deike-Hofmann, MD, *广 Christoph Kleinschnitz, MD,\# \\ Heinz-Peter Schlemmer, MD, PhD, † Michael Forsting, MD, * Werner Paulus, MD,// Louise F. Martin, DVM, ** \\ Christoph van Thriel, PhD, †† Uwe Karst, PhD, $\S$ and Astrid Jeibmann, MD, PhD/l
}

\begin{abstract}
Objectives: In recent years, complaints of patients about burning pain in arms and legs after the injection of gadolinium-based contrast agents (GBCAs) have been reported. In the current study, we investigated changes of small fibers in the epidermis as a potential cause of the patient complaints in a mouse model. Methods: Six groups of 8 mice were intravenously injected with either a macrocyclic GBCA (gadoteridol, gadoterate meglumine, gadobutrol), a linear GBCA (gadodiamide or gadobenate dimeglumine) $(1 \mathrm{mmol} / \mathrm{kg}$ body weight), or saline $(\mathrm{NaCl} \mathrm{0.9 \% ).} \mathrm{Four} \mathrm{weeks} \mathrm{after} \mathrm{injection,} \mathrm{animals} \mathrm{were} \mathrm{euthanized,} \mathrm{and} \mathrm{footpads}$ were assessed using immunofluorescence staining. Intraepidermal nerve fiber density (IENFD) was calculated, and the median number of terminal axonal swellings (TASs) per IENFD was determined.

Results: Nonparametric Wilcoxon signed-rank test revealed significantly lower IENFDs for all GBCAs compared with the control group $(P<0.0001)$ with the linear GBCAs showing significantly lower IENFDs than the macrocyclic GBCAs $(P<0.0001)$. The linear GBCAs presented significantly more TAS per IENFD than the control group $(P<0.0001)$, whereas no significant increase of TAS per IENFD compared with the control group was found for macrocyclic GBCAs $(P<0.237)$. Interpretation: It is unclear whether or at what dosage the decrease of IENFDs and the increase of TAS per IENFD found in the current animal model will appear in humans and if it translates into clinical symptoms. However, given the highly significant findings of the current study, more research in this field is required.
\end{abstract}

Key Words: small fiber neuropathy, gadolinium, GBCAs

(Invest Radiol 2020;55: 473-480)

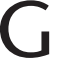
adolinium-based contrast agents (GBCAs) have become an indispensable part of the repertoire of radiological examinations. It is estimated that about 50 million doses of GBCAs are injected annually worldwide and that more than 500 million doses of GBCAs have been applied since their introduction in $1988 .{ }^{1}$ Generally, the safety profile of GBCAs is regarded as excellent, and the adverse effects are mostly mild, whereas severe adverse effects are very rare. ${ }^{2}$

Received for publication February 2, 2020; and accepted for publication, after revision, March 9, 2020

From the *Department of Diagnostic and Interventional Radiology and Neuroradiology, University Hospital Essen, University Duisburg-Essen, Essen; $†$ Department of Neuroradiology, University Hospital Bonn, Bonn, Germany; $₫$ Diagnostic Imaging Research Unit, Clinic for Diagnostic Imaging, University of Zurich, Zurich, Switzerland; §Institute of Inorganic and Analytical Chemistry, University of Münster; |Institute of Neuropathology, University Hospital Münster, Münster, Germany; Institute of Neuropathology, Justus Liebig University Giessen, Giessen; \#Department of Radiology, German Cancer Research Center, Heidelberg; **Neurology Clinic, University Hospital Essen, Essen, Germany; †'Institute of Laboratory Animal Science, Vetsuisse Faculty, University of Zurich, Zurich, Switzerland; and $\dagger$ †If A Do-Leibniz Research Centre for Working Environment and Human Factors, Dortmund, German.

Correspondence to: Alexander Radbruch, MD, JD, Department of Diagnostic and Interventional Radiology and Neuroradiology, University Hospital Essen, University Duisburg-Essen, Hufelandstraße 55, 45147 Essen, Germany. E-mail: alexander.radbruch@uk-essen.de.

Alexander Radbruch and Henning Richter contributed equally.

Conflicts of interest and sources of funding: none declared.

Copyright $(C) 2020$ Wolters Kluwer Health, Inc. All rights reserved.

ISSN: 0020-9996/20/5508-0473

DOI: $10.1097 /$ RLI.0000000000000677
Free gadolinium is toxic; therefore, it needs to be bound in a chelate for application in patients. ${ }^{3}$ According to the used chelate, the marketed GBCAs can be divided into linear GBCAs, in which the chelate partially encloses the gadolinium, and macrocyclic GBCAs, in which the gadolinium is completely enclosed by the chelate in the form of a cage. ${ }^{4,5}$ Macrocyclic GBCAs are more stable than linear GBCAs, and in vitro experiments in human serum revealed a gadolinium release of up to $20 \%$ for nonionic linear GBCAs, up to $2 \%$ for ionic linear GBCAs, and no gadolinium release above the limit of quantification (LOQ) for the macrocyclic GBCAs. ${ }^{6}$

In 2006, a correlation between the administration of GBCAs and nephrogenic systemic fibrosis (NSF) in patients with impaired kidney function was shown. ${ }^{7}$ Although the pathomechanism of NSF is not fully elucidated, the most common hypothesis is that gadolinium is released in vivo from the $\mathrm{GBCAs},{ }^{8}$ which is facilitated by the reduced clearance in patients with impaired kidney function. ${ }^{9}$ Subsequently, the released gadolinium is supposed to precipitate as insoluble gadolinium phosphate in the skin and ultimately leads to activation of circulating fibrocytes and a fibrotic reaction. ${ }^{10}$ The majority of unconfounded cases of NSF have been reported for linear GBCAs with the highest number of NSF cases per injection for the least stable nonionic GBCA gadodiamide, whereas there is an ongoing debate if macrocyclic GBCAs can cause NSF. ${ }^{11}$ Through consequent application of updated guidelines that restricted the use of GBCAs in patients with impaired kidney function as well as the preferred use of the more stable macrocyclic GBCAs for these patients, ${ }^{12,13}$ no new cases of NSF were reported since mid 2009. ${ }^{14}$

In 2015, it was shown that hyperintensities on MRI scans of the brain - first and foremost in the dentate nucleus - are caused by deposited gadolinium when linear GBCAs are injected. ${ }^{15,16}$ The currently prevailing theory assumes that linear GBCAs partially dechelate during the excretion process through the glymphatic system and potentially remain in the brain, whereas macrocyclic GBCAs are continuously washed out as intact chelate. ${ }^{17-21}$ Although a recent in vitro study reported mitochondrial toxicity and cell death in human neurons, especially for the least stable agents, ${ }^{22,23}$ the clinical significance of the gadolinium depositions in the brain is still under debate. ${ }^{18}$ However, it was hypothesized in the course of the renewed debate that injection of GBCAs in otherwise healthy patients might cause "various findings similar, but less severe than found in NSF." 24 The initial publication by Semelka et al was criticized due to the experimental design that did not include a control group, was not blinded, and was based on a patient survey. ${ }^{25}$ However, the proposed symptoms have been widely debated in patient forums and in the media ever since, and among the frequently described symptoms is burning skin pain, typically in the lower arms and lower legs. ${ }^{26}$

A disease that is typically associated with burning pain in the lower arms and legs is small fiber neuropathy (SFN). Small fiber neuropathy is a heterogeneous group of disorders affecting thinly myelinated $\mathrm{A} \delta$-fibers and unmyelinated $\mathrm{C}$-fibers. Although multiple causes of small nerve fiber degeneration have been reported, the cause of SFN remains unknown in up to $50 \%$ of cases. ${ }^{27}$ Diagnostic criterion 
standard to identify SFN is a skin biopsy in which the intraepidermal nerve fiber density (IENFD) is determined. ${ }^{28}$

In the current study, we assessed a potential correlation between the application of GBCAs and the IENFD in a mouse model. As a potential additional marker for damage of small fibers, the appearance of terminal axonal swellings (TASs) was assessed.

\section{MATERIALS AND METHODS}

Forty-eight female mice aged 24- to 31-day-old (RjOrl:SWISS) were included (Janvier; Elevage Le Genest, France) and randomly subdivided into 6 groups of 8 animals and housed in a conventional facility. Animals were injected with either the linear GBCAs (gadodiamide or gadobenate dimeglumine), macrocyclic GBCAs (gadobutrol, gadoteridol, or gadoterate meglumine), or with saline $(\mathrm{NaCl}$ $0.9 \%$ ) (Table 1). Injections of GBCAs were performed once intravenously at a dose of $1 \mathrm{mmol} / \mathrm{kg}$ body weight (BW) into the lateral tail vein. The control group received injections of saline according to the same study protocol ( $2 \mathrm{~mL} / \mathrm{kg}$, intravenous). The application within the groups was blinded and randomized. The study was performed at the Vetsuisse Faculty of the University of Zurich and was approved by the Cantonal Veterinary Office (animal permission number: ZH029/19). All analyses mentioned in the following sections were conducted blinded.

\section{Killing, Tissue Harvesting, and Sample Preparation}

All animals were euthanized by carbon dioxide inhalation 4 weeks after injection. Experiments were carried out according to the AVMA Guidelines for the Euthanasia of Animals. ${ }^{29}$ Death of the animals was confirmed by the arrest of the heartbeat and respiration. Tissue of both hind paws and both front paws as well as of the back was harvested immediately after the killing of the animals. After dissection, the tissues were stored at $-80^{\circ} \mathrm{C}$ or fixed in Zamboni's fixative (Morphisto $\mathrm{GmbH}$, Frankfurt am Main, Germany) according to the respective purposes.

\section{Sample Preparation for Total Gadolinium Quantification}

For total gadolinium quantification, mice back skin was shaved, dissected, and afterwards stored at $-80^{\circ} \mathrm{C}$ before homogenization via sectioning. For digestion, $500 \mu \mathrm{L}$ of concentrated nitric acid (Merck Chemicals GmbH, Darmstadt, Germany) and $100 \mu \mathrm{L}$ of $35 \%$ (wt/wt) hydrogen peroxide (Acros Organics, Geel, Belgium) were added to $0.05 \mathrm{~g}$ of each sample. Afterwards, the samples were heated to $80^{\circ} \mathrm{C}$ until fully digested. Finally, the samples were diluted, and rhodium inductively coupled plasma-mass spectrometry (ICP-MS) standard (SCP Science, Baie-D'Urfe, Quebec, Canada) was added as an internal standard. All samples were prepared in triplicate.

\section{Total Gadolinium Quantification}

The prepared digests were analyzed using an iCAP TQ ICP-MS (Thermo Fisher Scientific, Waltham, MA) in combination with an ASX-560 autosampler (Teledyne CETAC Technologies, Omaha, NE). The individual triplicate samples were analyzed 5 times each. Quantification was performed using a matrix matched external calibration in a range from $0 \mathrm{pg} / \mathrm{g}$ to $25 \mathrm{ng} / \mathrm{g}$ consisting of 12 standards. The standards were prepared by dilution of a $1000 \mu \mathrm{g} / \mathrm{g}$ gadolinium ICP-MS standard (Fluka Analytical, St Gallen, Switzerland). Again, rhodium was used as an internal standard.

The relative standard deviation (RSD) between 5 repetitive analyses of each individual triplicate sample was below 5\% for all digested samples, thus confirming the homogeneity of these samples. The average RSD between the triplicate samples of $10 \% \pm 11 \%$ is reasonable, considering the heterogeneous distribution of gadolinium in the biological sample. Due to biological variations between the animals, an average $\mathrm{RSD}$ of $43 \% \pm 16 \%$ was obtained for the distribution of the gadolinium concentration over all groups.

\section{Standard Preparation for LA-ICP-MS}

For calibration, 7 matrix-matched gelatin standards in a concentration range from $0 \mathrm{ng} / \mathrm{g}$ to $1 \mu \mathrm{g} / \mathrm{g}$ were created. For this purpose, a $1000-\mu \mathrm{g} / \mathrm{g}$ gadolinium ICP-MS standard (Fluka Analytical, St Gallen, Switzerland) was diluted and combined with gelatin of highest purity (Grüssing $\mathrm{GmbH}$, Filsum, Germany), resulting in standards with a gelatin content of $10 \%(\mathrm{wt} / \mathrm{wt})$. The suspension was homogenized by heating the standards to a temperature of $50^{\circ} \mathrm{C}$ and the usage of a vortex mixer. Afterwards, $10-\mu \mathrm{m}$-thin slices of each standard were prepared via a CryoStar NX70 Cryostat (Thermo Fisher Scientific, Waltham, MA). The thin sections of the standards were subsequently mounted onto an object slide.

The gadolinium concentration in the gelatin standards was confirmed by ICP-TQMS (iCAP TQ; Thermo Fisher Scientific, Waltham, $\mathrm{MA}$ ) analysis of the digested standards. For digestion, $500 \mu \mathrm{L}$ concentrated nitric acid (Merck Chemicals GmbH, Darmstadt, Germany) and $100 \mu \mathrm{L} \mathrm{35 \%}$ (wt/wt) hydrogen peroxide (Acros Organics, Geel, Belgium) were added to approximately $50 \mathrm{mg}$ of each gelatin standard. The mixture was then heated to a temperature of $70^{\circ} \mathrm{C}$ until complete digestion. Afterwards, the digests were diluted and a rhodium ICP-MS standard (SCP Science, Baie-D'Urfe, Quebec, Canada) was added as an internal standard. The concentration was determined using an external gadolinium calibration in a range from $0 \mathrm{pg} / \mathrm{g}$ to $25 \mathrm{ng} / \mathrm{g}$ consisting of 12 standards. Again, rhodium was used as an internal standard.

\section{Sample Preparation for LA-ICP-MS Measurements}

For LA-ICP-MS analysis, footpads of the front paws were excised and placed on a piece of cork using Tissue-Tek O.C.T. Compound (Sakura Finetek GmbH, Staufen, Germany). Thin sections of $10-\mu \mathrm{m}$

TABLE 1. Investigated Gadolinium-Based Contrast Agents

\begin{tabular}{|c|c|c|c|c|c|c|}
\hline & Control & Gadodiamide & Gadobenate & Gadoterate & Gadoteridol & Gadobutrol \\
\hline Commercial name & & Omniscan & MultiHance & Dotarem & ProHance & Gadovist \\
\hline Chemical form & & Linear, nonionic & Linear, ionic & Macrocyclic & Macrocyclic & Macrocyclic \\
\hline Amount of released gadolinium found by Frenzel et $\mathrm{al}^{6}$ & & $20 \%$ & $1.9 \%$ & $<$ LOQ & $<\mathrm{LOQ}$ & $<\mathrm{LOQ}$ \\
\hline Gadolinium content in back skin $\pm \mathrm{SD}, \mathrm{ng} / \mathrm{g}$ & $2.7 \pm 0.4$ & $203 \pm 116$ & $67 \pm 24$ & $20 \pm 9$ & $32 \pm 18$ & $28 \pm 14$ \\
\hline IENFD, $\mathrm{mm}^{-1}$ & $21.10 \pm 4.41$ & $10.19 \pm 2.27$ & $10.00 \pm 0.86$ & $13.08 \pm 2.58$ & $12.76 \pm 1.68$ & $11.89 \pm 1.24$ \\
\hline TAS, no. cases & $0 / 8$ & $8 / 8$ & $6 / 8$ & $2 / 8$ & $2 / 8$ & $3 / 8$ \\
\hline $\mathrm{TAS} / \mathrm{IENFD} \pm \mathrm{SEM}, \mathrm{mm}$ & $0.000 \pm 0.000$ & $0.182 \pm 0.159$ & $0.109 \pm 0.189$ & $0.008 \pm 0.023$ & $0.004 \pm 0.012$ & $0.025 \pm 0.049$ \\
\hline
\end{tabular}

The amount of gadolinium released in the study by Frenzel et $\mathrm{al}^{6}$ (in vitro study in patient serum at $37^{\circ} \mathrm{C}$ after 2 weeks) characterizes the stability of the GBCAs with gadodiamide being the least stable GBCA. Summary of results for gadolinium content of back skin, intraepidermal nerve fiber density, number of animals with TASs, and TAS/IENFD.

LOQ, limit of quantification 
thickness were cut with a cryotome, and 8 sections were mounted on a microscopic glass slides. Before the ablation process, microscopic images were recorded with a BZ-9000 inverted fluorescence/bright field microscope (Keyence, Osaka, Japan).

\section{LA-ICP-MS Measurements}

For elemental mapping of the gadolinium distribution, the combination of a laser ablation (LA) instrument model NWRimage (ESI, Bozeman, MT) and an ICP-MS instrument model iCAP TQ (Thermo Fisher Scientific, Waltham, MA) was used. For ablation, a spot size of $20 \mu \mathrm{m}$ and a stage speed of $100 \mu \mathrm{m} / \mathrm{s}$ as well as a shot frequency of $100 \mathrm{~Hz}$ were chosen. Laser energy was optimized for each individual sample to allow for quantitative ablation of the sample with minor ablation of the microscopic slide. Ablated particles were washed out by a constant helium flow of $1100 \mathrm{~mL} / \mathrm{min}$. For more efficient transportation, a daily tuned argon flow was added in the Dual Concentric Injector (ESI, Bozeman, MT) of the ICP-MS system. Argon plasma with an RF power of $1550 \mathrm{~W}$ was used for the ionization. The ionized analytes were guided through a nickel interface and were analyzed by a triple quadrupole mass spectrometer (TQ-MS) setup.

For quantification, the matrix-matched gadolinium gelatin standards were used. On each standard, 11 lines with an ablation time of 20 seconds per line were ablated with the same parameters as used for the sample. The first line of each measurement was discarded for consistency reasons. All data evaluation was performed using the software ImaJar (developed by Robin Schmidt, Münster, Germany). The LOQ was defined as 10 times the standard deviation of the evaluated pixels of the blank sample divided by the calibration slope. To account for the limited quantification possibilities, although still illustrating the full dataset of a quantified image, all data points below the LOQ are shown in a grayscale.

\section{Fluorescence Immunohistochemistry}

Footpads taken from all animals were fixed in Zamboni solution, washed 3 times with $0.1 \mathrm{M}$ of Phosphate-buffered saline (PBS), and stored at $4{ }^{\circ} \mathrm{C}$. After medium change to $10 \%$ sucrose and incubation over 24 hours, 50- $\mu$ m-thick cryosections were prepared and stored in $0.1 \%$ Triton PBS. After 3 times washing for 5 minutes in $0.1 \%$ Triton PBS, immunofluorescence staining was performed at free-floating sections with a polyclonal antibody against the neuron-specific hydrolase protein gene product (PGP) 9.5 (rabbit polyclonal, 1:1000, \#516-3344; Zytomed Systems GmbH, Berlin, Germany) and incubated overnight.

After 3 times washing for 15 minutes in $0.1 \%$ Triton PBS, sections were stained with Alexa 488 (goat anti-rabbit 1:1000, \#A11008; Thermo Fisher Scientific, Waltham, MA) and incubated at room temperature for 2 hours. After 3 times washing for 15 minutes in $0.1 \%$ Triton PBS, the sections were mounted with Fluoromount-G with DAPI (\#00-4959-52; Thermo Fisher Scientific, Waltham, MA) and stored at $4^{\circ} \mathrm{C}$ in the dark until evaluation.

\section{Quantification of Intraepidermal Nerve Fiber Density and Terminal Axonal Swellings}

Intraepidermal nerve fiber density was analyzed using a BX50 microscope (Olympus Europe, Hamburg, Germany) with Fluorescein isothiocyanate (FITC) filter cube and $40 \times$ objective. The IENFD was calculated for a triplicate of all samples and documented as the mean of IENF per millimeter based on the respective triplicate. We scanned for subepidermal and intraepidermal nerves through 2 planes: horizontally along the epidermal-dermal border and vertically by focusing through the thickness of the section in order to detect each nerve branch and its course as suggested by guidelines. ${ }^{28}$ Two observers (A.J., H.R.) blinded to the injected GBCA counted in each section the total number of IENFs clearly penetrating the basement membrane following established criteria using the software ImageJ (ImageJ for Mac OS X, version $1.51[100])$. The IENFD was calculated as mean number of nerve fibers per millimeter epidermal length (IENF per mm). In addition, terminal axonal swellings (TASs) were investigated in 3 sections per animal. Animals with TAS were identified and subjected to quantification. In a second step, the number of TAS per section was calculated and divided by IENFD of the respective section. Results were plotted using GraphPad Prism 8 (GraphPad Software, San Diego, CA).
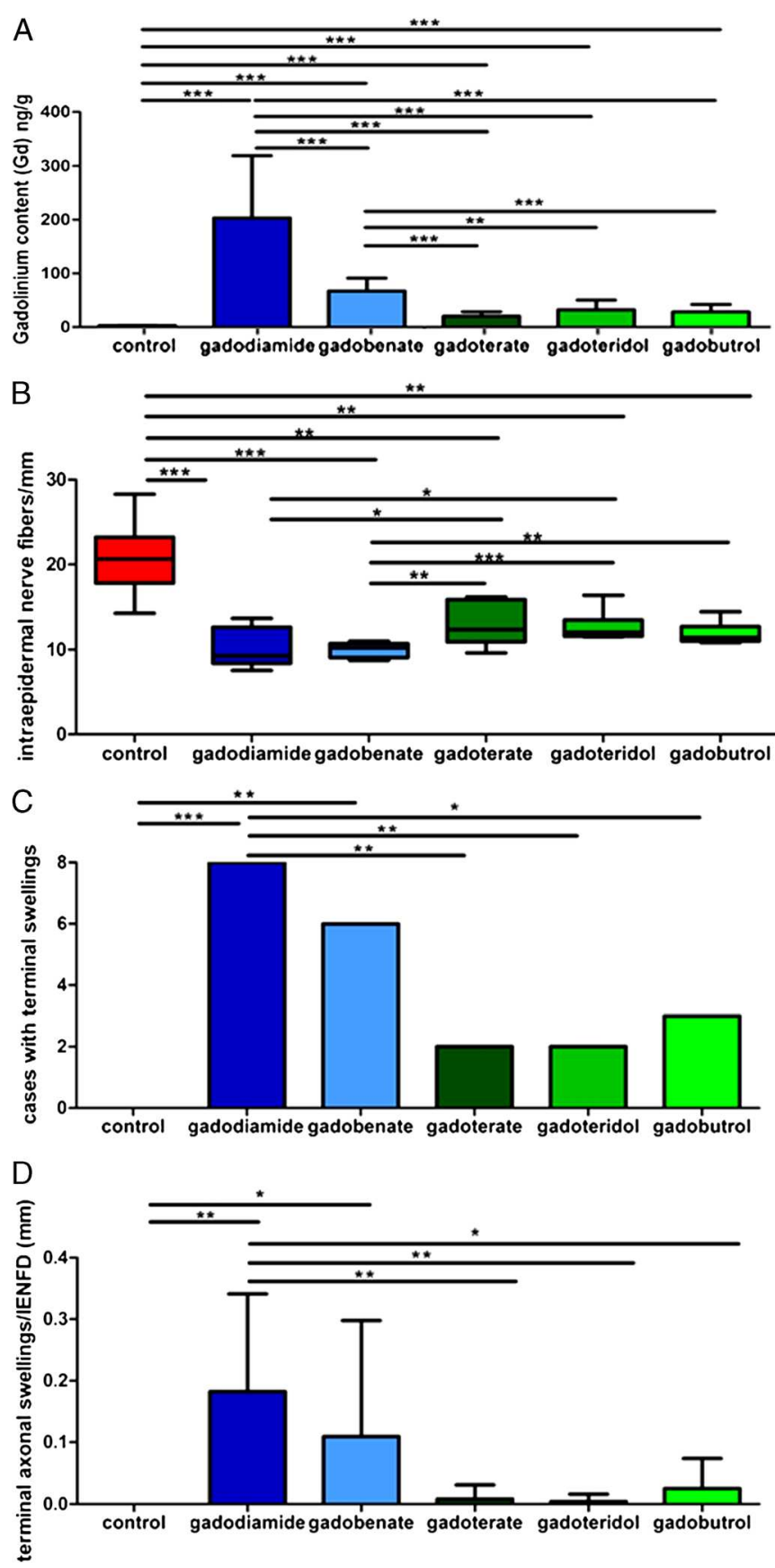

FIGURE 1. Overview of gadolinium content (A), intraepidermal nerve fiber density (IENFD) (B), cases with TASs (C), and TASs/IENFD (D). Linear GBCAs gadodiamide (dark blue) and gadobenate dimeglumine (light blue) showed a significantly higher amount of gadolinium in the back skin of the mice compared with the macrocyclic GBCAs (shades of green). In contrast, linear GBCAs showed a significantly higher decrease of IENFD $\left(\mathrm{mm}^{-1}\right)(B)$ and a significantly higher increase of TAS $(C)$ and TAS/IENFD (mm) (D). * $*<0.05 ; * * P<0.01 ; * * * P<0.001$. 
For illustration of intraepidermal nerve fibers and TAS (Figs. 2F, G, I, J), an Axio Imager M2 with a laser scanning microscope LSM 700 confocal unit (Zeiss) was used (lenses: $10 \times$ Plan Apo, numerical aperture $=0.45 ; 40 \times$ Plan Apo, numerical aperture = 1.4). Zen 2009 software (Zeiss) was used by generating a maximum intensity projection of the z-planes.

\section{Statistical Analysis}

Statistical analyses were performed using the SPSS software (Version 25 for Mac, 64-bit edition; IBM, Chicago, IL). Descriptive analyses were performed for continuous variables. Due to the small number of animals, the data were analyzed as nonparametric. For testing significance between group distributions, a Kruskal-Wallis test between more than 2 groups was used, followed by a Wilcoxon rank sum test (Mann-Whitney $U$ test) between 2 independent measurements. Interobserver reliability for IENF evaluation was analyzed using an intraclass correlation coefficient. The level of significance was set at $P<0.05$.

\section{RESULTS}

\section{Total Gadolinium Concentration in the Back Skin of} Mice Is Highest for the Linear Nonionic GBCA

\section{Gadodiamide and Lowest for the Macrocyclic GBCAs}

The small size of the paws and accordingly the limited amount of tissue for sample preparation impeded a gadolinium quantification directly in the paw (with the exception for the linear nonionic GBCA gadodiamide that exhibited the highest amounts of gadolinium). Rather, we assessed tissue of the back skin as a substitute, because larger amounts of tissue were available for this region. Four weeks after injection, the highest concentration of gadolinium in the back skin was found for gadodiamide $(203 \pm 116 \mathrm{ng} / \mathrm{g})$, which exhibited significantly more gadolinium than all other assessed GBCAs. The linear ionic GBCA gadobenate $(67 \pm 24 \mathrm{ng} / \mathrm{g})$ exhibited significantly more gadolinium than all 3 macrocyclic GBCAs, which did not differ significantly within their group (20 $\pm 9 \mathrm{ng} / \mathrm{g} ; 32 \pm 18 \mathrm{ng} / \mathrm{g} ; 28 \pm 14 \mathrm{ng} / \mathrm{g}$ ). The calculated average concentrations of each group are illustrated in Figure 1A and Table 1.
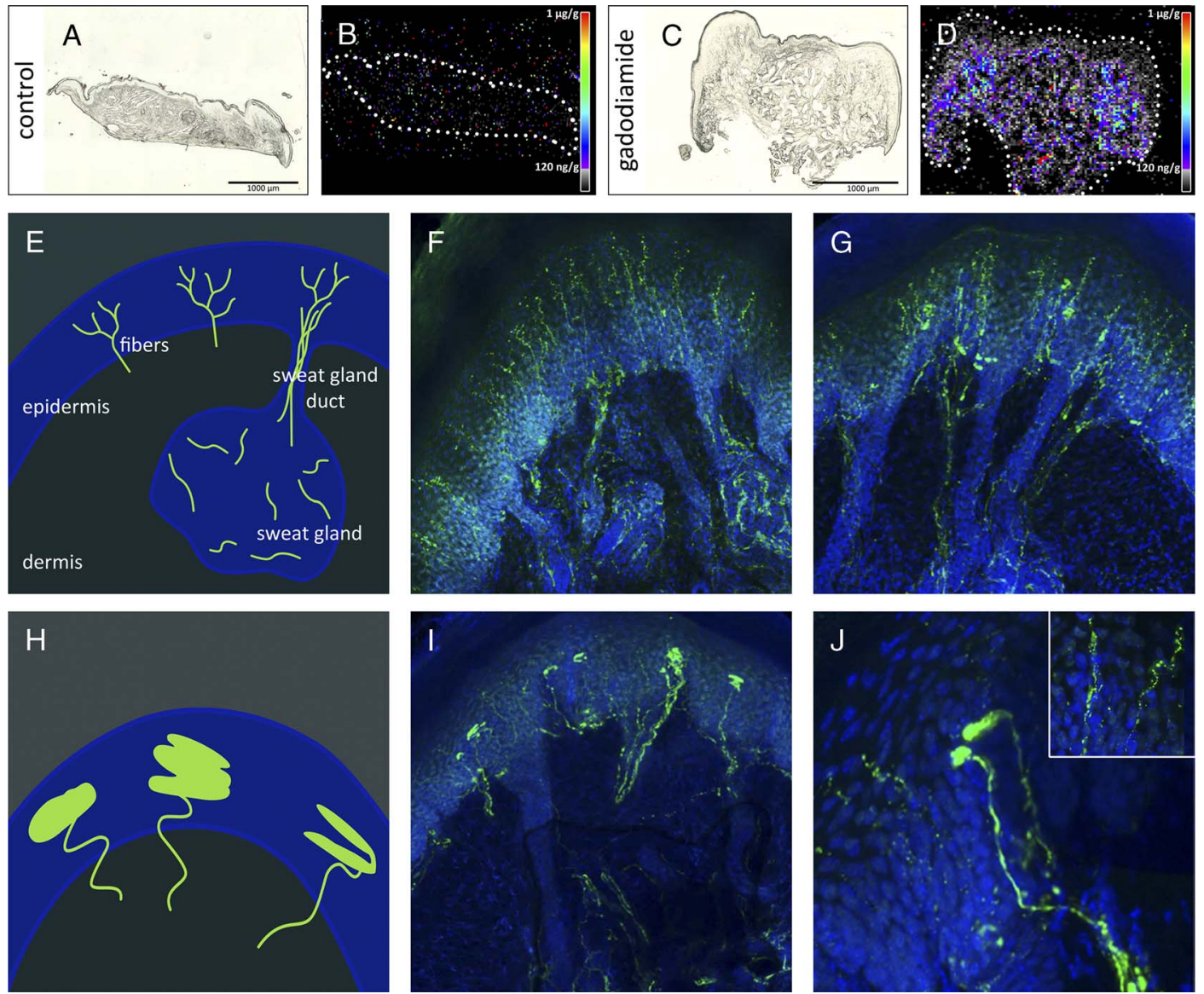

FIGURE 2. Gadolinium content and distribution in foot pads determined by laser ablation-inductively coupled plasma-mass spectrometry (LA-ICP-MS) ( $C$ and $D$ ) in comparison to control (A and B) and changes of small fibers, stained for PGP9.5 (IENF) (E-G, I) and TAS (H-J). LA-ICP-MS analyses of foot pads (marked by dotted lines) treated with gadodiamide (C and D) show gadolinium in the dermis of the footpad in comparison to control foot pad treated with saline (A and B). Schematic overview of the dermis and epidermis with small fibers (depicted in green) as well as sweat glands and sweat gland ducts (E). Slightly reduced IENFD after administration of gadobutrol (G) and strongly decreased IENFD after administration of gadodiamide (I) in comparison to control (F). Schematic overview of the epidermis with TAS (H). Terminal axonal swellings are clearly detectable after gadodiamide administration (J) in comparison to control (inset). The highest TAS/IENFD that was found for gadodiamide caused a clear visual pattern that allowed a direct identification of the animals injected with gadodiamide based on the first visual inspection. 
TABLE 2. Quantification of Intraepidermal Nerve Fibers

\begin{tabular}{|c|c|c|c|c|c|c|}
\hline & Control & Gadodiamide & Gadobenate & Gadoterate & Gadoteridol & Gadobutrol \\
\hline Control & & 0.000 & 0.000 & 0.002 & 0.002 & 0.001 \\
\hline Gadodiamide & 0.000 & & 0.878 & 0.038 & 0.050 & 0.130 \\
\hline Gadobenate & 0.000 & 0.878 & & 0.007 & 0.000 & 0.001 \\
\hline Gadoterate & 0.002 & 0.038 & 0.007 & & 1.000 & 0.382 \\
\hline Gadoteridol & 0.002 & 0.050 & 0.000 & 1.000 & & 0.105 \\
\hline Gadobutrol & 0.001 & 0.130 & 0.001 & 0.382 & 0.105 & \\
\hline
\end{tabular}

$P$ values of nonparametrical Wilcoxon rank sum test (Mann-Whitney $U$ test) between linear GBCAs (gadodiamide, gadobenate dimeglumine), macrocyclic GBCAs (gadoteridol, gadoterate meglumine, gadobutrol), and control $(\mathrm{NaCl})$. Level of significance, $P<0.05$.

\section{Gadolinium Detection Is Restricted to the Dermis of Mice Footpads Determined by LA-ICP-MS in Mice Injected With Gadodiamide}

Under the selected conditions, the found LOQ of $120 \mathrm{ng} / \mathrm{g}$ for gadolinium in LA-ICP-MS exceeded the average gadolinium concentration in the back skin for all mice except those from the gadodiamide group, which was $203 \pm 116 \mathrm{ng} / \mathrm{g}$. Therefore, only gadodiamide could be subjected to footpads gadolinium quantification via LA-ICP-MS. Gadolinium was detected exclusively in the dermis, whereas no gadolinium deposition was found in the epidermis (Fig. 2).

\section{Intraepidermal Nerve Fiber Density Is Significantly Reduced After Administration of GBCAs}

The IENFD was determined by 2 observers (A.J. and H.R.), blinded to the study groups, according to standard procedures. The interobserver agreement was substantial (intraclass correlation coefficient, $0.795 ; 95 \%$ confidence interval, $0.633-0.886$ ). The IENFD was significantly reduced 4 weeks after injection with GBCAs in comparison to controls $(P<0.0001$; Fig. 1B). Although IENFD differed significantly when comparing linear $(P<0.0001)$ with macrocyclic GBCAs, with a more pronounced IENFD reduction after injection of linear GBCAs, IENFDs did not significantly differ within the chemical GBCA groups (Fig. 1B, Tables 1-3).

\section{Administration of GBCAs Results in Terminal Axonal Swellings of Small Fibers in All Mice Injected With Gadodiamide, Whereas No Animals Injected With Saline Exhibited TAS}

Beyond the quantification of IENFD, sections stained with the neuronal marker PGP9.5 were subjected to further analysis. Conglomerates at the terminal endings of intraepidermal small fibers, positive for PGP9.5 (Fig. 2H), were detected and described as TAS, here illustrated for animals after application of gadodiamide (Figs. 2I,J).

Terminal axonal swellings were visible exclusively after GBCA administration, whereas no TAS displayed in the control group (Fig. 1C). Terminal axonal swellings were apparent in all 8 cases after application of gadodiamide and in 6 of 8 cases injected with gadobenate (Fig. 1C), whereas only 2 or 3 out of 8 animals exhibited TAS when macrocyclic GBCAs were injected (for further details, see Tables 1, 4, and 5).

To further analyze TAS, we subjected PGP9.5-stained specimens to quantitative analysis and assessed the number of TAS per IENFD of the respective specimen (Fig. 1D). After administration of gadodiamide, significantly more TAS per IENFD were detected in comparison to the control group $(P<0.01)$ and in comparison to all macrocyclic GBCAs (gadoterate, gadoteridol, and gadobutrol) $(P<0.01, P<0.01$, and $P<0.05)$, whereas no significant difference could be detected between the linear ionic GBCA gadobenate and the macrocyclic GBCAs (for further details, see Tables 1, 6, and 7).

\section{DISCUSSION}

In the current study, we found a significant reduction of IENFD in the footpad of mice for all GBCAs 4 weeks after a single injection of $1 \mathrm{mmol} / \mathrm{kg} \mathrm{BW}$. There was a significantly larger decrease of IENFD for the linear GBCAs compared with the macrocyclic GBCAs. Moreover, we found a significant increase of TAS/IENFD for the linear GBCAs, whereas only a trend without significance was found for the macrocyclic GBCAs. The gadolinium concentrations found in the back skin of the animals are in agreement with previous studies showing a correlation between the complex stability of GBCAs (with the linear nonionic being the least stable and the macrocyclic being the most stable GBCAs) and the amount of residual gadolinium in the skin. ${ }^{30,31}$ Moreover, only the least stable nonionic linear GBCA gadodiamide exceeded the LOQ in the LA-ICP-MS in the footpad.

To the best of our knowledge, the current study is the first to investigate a correlation between small fiber degeneration and GBCA exposure. Although the debate on NSF started with reports on skin pathologies and pain has been reported as one of the major symptoms of NSF, little attention has been paid on a potential involvement of small fibers in the skin. In contrast, research focused mainly on elucidating the cascade that ends in the activation of fibroblasts and is likely triggered by the release of gadolinium from the GBCAs. ${ }^{10}$

\section{Potential Mechanism of GBCA-Mediated IENFD Reduction and TAS Increase}

The pathomechanism that causes the decrease of IENFD and the increase of TAS is currently unclear. Generally, toxic effects of GBCAs can be mediated by either the intact chelate, the ligand, or the released gadolinium. ${ }^{23}$ In the current study, no speciation analyses were conducted. However, previous animal studies provided evidence for a partial dechelation and a gadolinium release of the linear GBCAs, whereas no gadolinium release above the LOQ has been shown for the macrocyclic GBCAs in vivo so far. ${ }^{19}$ The theory of a partial dechelation of the linear GBCAs also explains the increased levels of gadolinium that has been shown in the current study as well as in previous animal studies. ${ }^{19,30,31}$ Although the intact chelate of the macrocyclic GBCAs does not bind to other partners and is washed out from the skin over time, the released gadolinium from the linear GBCAs might result in potentially

TABLE 3. Quantification of Intraepidermal Nerve Fibers

\begin{tabular}{lccc}
\hline & Control & Linear & Macrocyclic \\
\hline Control & & 0.000 & 0.000 \\
Linear & 0.000 & & 0.000 \\
Macrocyclic & 0.000 & 0.000 & \\
\hline
\end{tabular}

$P$ values of nonparametrical Wilcoxon rank sum test between grouped GBCAs (linear, macrocyclic) and grouped controls. Level of significance, $P<0.05$. 
TABLE 4. Terminal Axonal Swelling-Dichotomous Analysis

\begin{tabular}{|c|c|c|c|c|c|c|}
\hline & Control & Gadodiamide & Gadobenate & Gadoterate & Gadoteridol & Gadobutrol \\
\hline Control & & 0.000 & 0.010 & 0.442 & 0.442 & 0.234 \\
\hline Gadodiamide & 0.000 & & 0.442 & 0.010 & 0.010 & 0.038 \\
\hline Gadobenate & 0.010 & 0.442 & & 0.105 & 0.105 & 0.234 \\
\hline Gadoterate & 0.442 & 0.010 & 0.105 & & 1.000 & 0.721 \\
\hline Gadoteridol & 0.442 & 0.010 & 0.105 & 1.000 & & 0.721 \\
\hline Gadobutrol & 0.234 & 0.038 & 0.234 & 0.721 & 0.721 & \\
\hline
\end{tabular}

$P$ values of nonparametrical Wilcoxon rank sum test between linear GBCAs (gadodiamide, gadobenate dimeglumine), macrocyclic GBCAs (gadoteridol, gadoterate meglumine, gadobutrol), and control ( $\mathrm{NaCl})$. Level of significance, $P<0.05$.

long-standing gadolinium deposition when it is bound to new partners (eg, phosphate or macromolecules; Fig. 3). ${ }^{19,30,31}$ Also, we chose the timeframe of 4 weeks in the current study because previous studies showed that the majority of macrocyclic GBCAs is washed out after this period. ${ }^{31}$

For the linear GBCAs, it might be speculated that the observed toxic effects could be mediated by a competitive binding of the released gadolinium-ion to calcium channels in the small fibers. ${ }^{32}$ However, this mechanism cannot explain the observed effects for the macrocyclic GBCAs, which must be mediated by the intact chelate. Notably, any toxic effect mediated by released gadolinium from the linear GBCAs might potentially be more problematic than toxic effects from the intact

TABLE 5. Terminal Axonal Swelling-Dichotomous Analysis

\begin{tabular}{lccc}
\hline & Control & Linear & Macrocyclic \\
\hline Control & & 0.000 & 0.237 \\
Linear & 0.000 & & 0.002 \\
Macrocyclic & 0.237 & 0.002 & \\
\hline
\end{tabular}

$P$ values of nonparametrical Wilcoxon rank sum test between grouped GBCAs (linear, macrocyclic) and grouped controls. Level of significance, $P<0.05$.

chelate because gadolinium bound to new partners might remain in the skin, whereas toxicity mediated by the intact complex disappears when the complex is washed out. In the current study, the potentially increased toxic effect of the least stable linear nonionic GBCA gadodiamide becomes apparent when the parameter TAS/IENFD is assessed. For gadodiamide, this ratio was 7.3 to 45.5 higher compared with the macrocyclic GBCAs, which resulted in a clear visual pattern of the stains that allowed a direct identification of the gadodiamide stains based on the first visual impression (Fig. 2).
Finally, toxic effects caused by either the intact chelate or the released gadolinium might first become apparent above a certain threshold. Based on the recommendations of the Food and Drug Administration, interspecies scaling for determination of the human equivalent dose (HED) should be based on body surface area scaling. ${ }^{33}$ According to this approach, the dosage applied in the current study of $1 \mathrm{mmol} / \mathrm{kg}$ BW results in an HED for an adult human of $0.081 \mathrm{mmol} / \mathrm{kg} \mathrm{BW}$, which is slightly below the clinically applied dosage of $0.1 \mathrm{mmol} / \mathrm{kg}$ BW used in humans. ${ }^{33}$ However, in recent years, the HED calculation based on the body surface area has been criticized as "oversimplistic." 34 Currently, it remains unclear if and at what dosage the findings of the current mouse study can be transferred to humans.

\section{Clinical Effect of IENFD Decrease and Axonal Swelling Increase}

Generally, SFN is a disorder of thinly myelinated A $\delta$ and unmyelinated $\mathrm{C}$ fibers that does not show abnormalities in motor and large sensory nerve fiber function at neurological examination, whereas hyperalgesia and allodynia frequently accompany nociceptive and temperature sensation loss. ${ }^{35}$ It needs to be stressed that the arm and leg pain some patients are complaining about after GBCA injection is neither clinically nor histopathologically assessed adequately. Hence, no conclusions about the origin of the reported pain can be drawn so far.

However, decreased IENFD has been shown to correlate with neuropathic pain in patients receiving chemotherapy. ${ }^{36,37}$ No comparable studies are available for the assessed TAS, and appearance of comparable alterations was mostly reported for acrylamide intoxication. ${ }^{38}$ At least the visual appearance of the TAS as a PGP9.5-positive conglomerate at the endings of small fibers does not make a clinical effect of this finding appear unlikely.

A possible argument against any severe effect of GBCAs causing neuropathic pain might be the large number of injections of GBCAs that have been applied to patients and the comparably low number of patients who complain of arm and leg pain after GBCA injections.

TABLE 6. Terminal Axonal Swelling-Quantitative Analysis

\begin{tabular}{|c|c|c|c|c|c|c|}
\hline & Control & Gadodiamide & Gadobenate & Gadoterate & Gadoteridol & Gadobutrol \\
\hline Control & & 0.004 & 0.038 & 0.350 & 0.350 & 0.198 \\
\hline Gadodiamide & 0.004 & & 0.279 & 0.009 & 0.005 & 0.016 \\
\hline Gadobenate & 0.038 & 0.279 & & 0.074 & 0.074 & 0.177 \\
\hline Gadoterate & 0.350 & 0.009 & 0.074 & & 0.927 & 0.515 \\
\hline Gadoteridol & 0.350 & 0.005 & 0.074 & 0.927 & & 0.515 \\
\hline Gadobutrol & 0.198 & 0.016 & 0.177 & 0.515 & 0.515 & \\
\hline
\end{tabular}

$P$ values of nonparametrical Wilcoxon rank sum test between linear GBCAs (gadodiamide, gadobenate dimeglumine), macrocyclic GBCAs (gadoteridol, gadoterate meglumine, gadobutrol), and control (NaCl). Level of significance, $P<0.05$. 
TABLE 7. Terminal Axonal Swelling-Quantitative Analysis

\begin{tabular}{lccc}
\hline & Control & Linear & Macrocyclic \\
\hline Control & & 0.013 & 0.532 \\
Linear & 0.013 & & 0.000 \\
Macrocyclic & 0.532 & 0.000 & \\
\hline
\end{tabular}

$P$ values of nonparametrical Wilcoxon rank sum test between grouped GBCAs (linear, macrocyclic) and grouped controls. Level of significance, $P<0.05$.

However, patients who get GBCA injections usually have confounding factors. Hence, it remains a conceivable scenario that in the past we wrongly classified neuropathic pain in patients as an adverse effect of therapies or accidents, although it might have been caused by the use of GBCAs. In this respect, we should remind ourselves that also the appearance of NSF remained unrecognized for almost 20 years.

\section{LIMITATIONS}

Limitations of the study need to be acknowledged. As highlighted previously, the transferability of the results from animal experiments to humans and especially the determination of the HED remain crucial unknown points. Another important limitation of the study is that we did not assess potential regeneration changes over time and hence cannot conclude if the decrease of IENFD and the TAS increase will be permanently or temporarily. Ultimately, we did not assess the exact pathomechanism of the IENFD decrease and the TAS increase.

Obvious next steps would be animal studies investigating the threshold concentration when a decrease of IENFD first becomes apparent as well as studies assessing serial injections of GBCAs. Again, it needs to be highlighted that serial injections of macrocyclic GBCAs might not cause any measurable gadolinium - or only minor gadolinium deposition - if the washout period is sufficiently long in between the injections, whereas in contrast the released gadolinium from the linear GBCAs might successively cumulate in the skin. ${ }^{18,31}$

Also, it needs to be emphasized that only 5 of the marketed GBCAs have been assessed in the current study, and future studies might include especially the GBCAs gadoversetamide and gadopentetate dimeglumine.

Finally, skin biopsy studies might be considered in patients with complaints after GBCA exposure, although potentially confounding factors will be a challenge.

We want to emphasize that one of the biggest dangers of the current debate on potential gadolinium toxicity might be the unreasonable decline of GBCAs in clinically indicated situations. ${ }^{18,39}$ Moreover, we are aware that the results of our study bear the potential to scare patients as well as treating radiologists. Hence, we urge the scientific and radiological community to treat the results with caution as long as no confirmatory studies in patients are available. In this respect, it is important
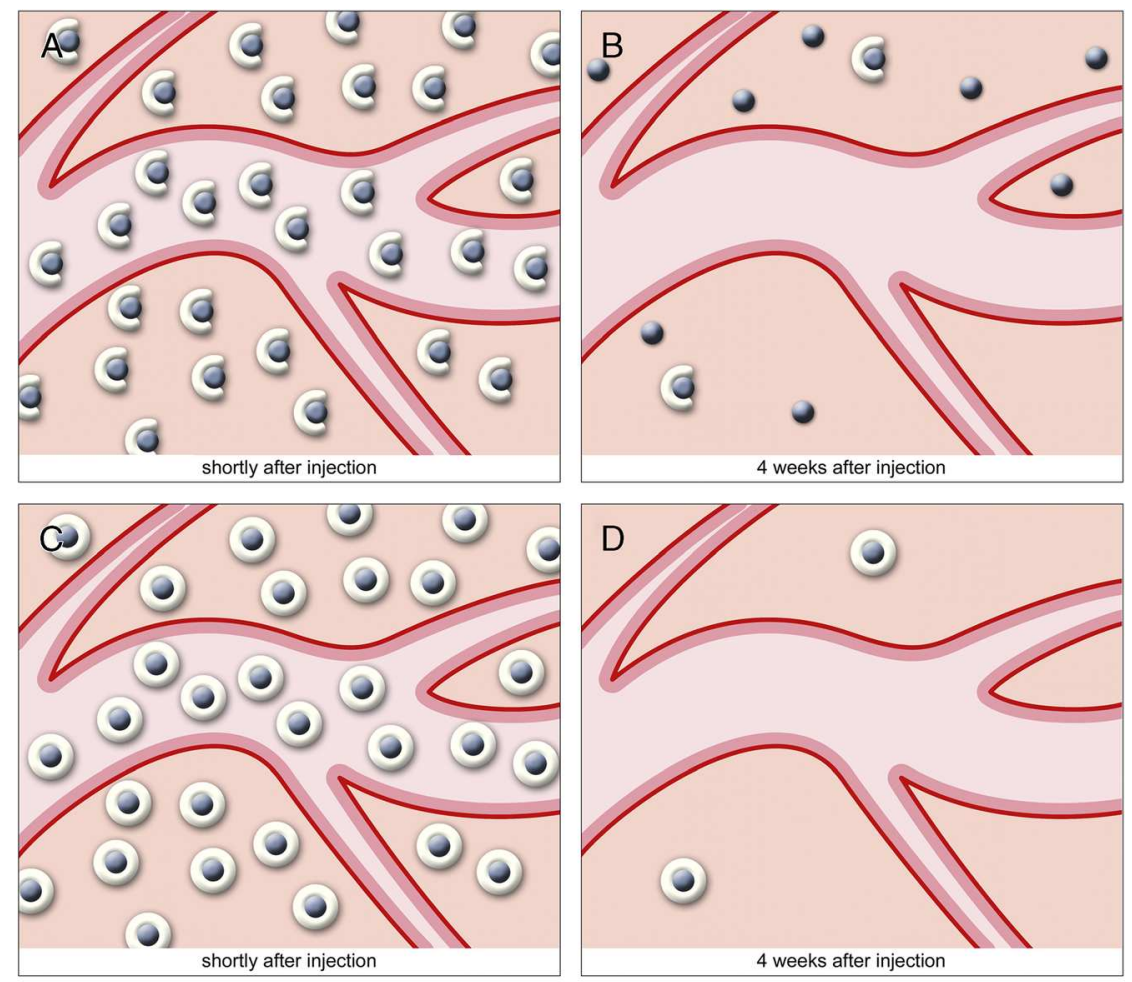

gadolinium

linear GBCA

macrocyclic GBCA

FIGURE 3. Hypothesis on local toxicity of GBCAs. Shortly after injection, high amounts of the intact chelate (linear GBCAs in A and macrocyclic GBCAs in C) pass through blood vessels in the dermis and get in the interstitium, where potential toxic effects can be mediated. The significantly higher amounts of gadolinium in the skin for the linear GBCAs found 4 weeks after injection in the current study might be explained by a partial dechelation of the less stable linear GBCAs (B), whereas no dechelation of macrocyclic GBCAs has been reported in vivo so far (D). The dechelated gadolinium might mediate increased toxicity on the small fibers, which could explain the higher decrease of IENFDs and the higher increase of TAS/IENFDs for the linear GBCAs compared with the macrocyclic GBCAs. 
that GBCAs need to be treated as any other medication, and a comprehensive risk-benefit analysis needs to be performed before injection of any GBCA. ${ }^{40}$ In March 2018, the European Medicines Agency concluded in a precautionary approach that this risk-benefit evaluation is no longer positive for the linear GBCAs and decided to withdraw them (apart from liver imaging and intra-articular injections) from the market. ${ }^{18}$ In contrast, the Food and Drug Administration decided to keep all GBCAs on the US market because no clinical correlates were proven for the gadolinium deposition. From a risk minimization point of view, the current study lends further support for the European approach. U1timately, the results of the current study should strengthen scientific projects that aim to reduce the applied amount of GBCAs or support the use of contrast agent-free techniques. ${ }^{41-43}$

\section{CONCLUSIONS}

In the current study, we found a significant decrease of IENFD 4 weeks after a single injection of all GBCAs $(1 \mathrm{mmol} / \mathrm{kg} \mathrm{BW})$ in the footpads of mice. The IENFD decrease was significantly higher after injection of linear compared with macrocyclic GBCAs. In addition, mice injected with linear GBCAs showed a significant increase of TAS per IENFD, whereas macrocyclic GBCAs showed a TAS per IENFD increase that did not reach the level of significance compared with controls. The clinical significance of these findings remains unclear.

\section{REFERENCES}

1. Runge VM. Commentary on T1-weighted hypersignal in the deep cerebellar nuclei after repeated administrations of gadolinium-based contrast agents in healthy rats: difference between linear and macrocyclic agents. Invest Radiol. 2015;50:481-482.

2. Matsumura T, Hayakawa M, Shimada F, et al. Safety of gadopentetate dimeglumine after 120 million administrations over 25 years of clinical use. Magn Reson Med Sci. 2013;12:297-304.

3. Idee JM, Port M, Raynal I, et al. Clinical and biological consequences of transmetallation induced by contrast agents for magnetic resonance imaging: a review. Fundam Clin Pharmacol. 2006;20:563-576.

4. Hao D, Ai T, Goerner F, et al. MRI contrast agents: basic chemistry and safety. J Magn Reson Imaging. 2012;36:1060-1071.

5. Idee JM, Port M, Robic C, et al. Role of thermodynamic and kinetic parameters in gadolinium chelate stability. J Magn Reson Imaging. 2009;30:1249-1258.

6. Frenzel T, Lengsfeld P, Schirmer H, et al. Stability of gadolinium-based magnetic resonance imaging contrast agents in human serum at 37 degrees C. Invest Radiol. 2008;43:817-828.

7. Grobner T. Gadolinium-a specific trigger for the development of nephrogenic fibrosing dermopathy and nephrogenic systemic fibrosis? Nephrol Dial Transplant. 2006;21:1104-1108.

8. Khurana A, Runge VM, Narayanan M, et al. Nephrogenic systemic fibrosis: a review of 6 cases temporally related to gadodiamide injection (Omniscan). Invest Radiol. 2007;42:139-145.

9. Thomsen HS, Morcos SK, Almen T, et al. Nephrogenic systemic fibrosis and gadolinium-based contrast media: updated ESUR contrast medium safety committee guidelines. Eur Radiol. 2013;23:307-318.

10. Gupta A, Shamseddin MK, Khaira A. Pathomechanisms of nephrogenic systemic fibrosis: new insights. Clin Exp Dermatol. 2011;36:763-778.

11. Endrikat J, Dohanish S, Schleyer N, et al. 10 years of Nephrogenic systemic fibrosis: a comprehensive analysis of nephrogenic systemic fibrosis reports received by a pharmaceutical company from 2006 to 2016. Invest Radiol. 2018;53:541-550.

12. Wang Y, Alkasab TK, Narin O, et al. Incidence of nephrogenic systemic fibrosis after adoption of restrictive gadolinium-based contrast agent guidelines. Radiology. 2011;260:105-111.

13. Bennett CL, Qureshi ZP, Sartor AO, et al. Gadolinium-induced nephrogenic systemic fibrosis: the rise and fall of an iatrogenic disease. Clin Kidney J. 2012;5:82-88.

14. Huckle JE, Altun E, Jay M, et al. Gadolinium deposition in humans: when did we learn that gadolinium was deposited in vivo? Invest Radiol. 2016;51:236-240.

15. Kanda T, Ishii K, Kawaguchi H, et al. High signal intensity in the dentate nucleus and globus pallidus on unenhanced T1-weighted MR images: relationship with increasing cumulative dose of a gadolinium-based contrast material. Radiology. 2014;270:834-841.
16. Radbruch A, Weberling LD, Kieslich PJ, et al. Gadolinium retention in the dentate nucleus and globus pallidus is dependent on the class of contrast agent. Radiology. 2015;275:783-791.

17. Deike-Hofmann K, Reuter J, Haase R, et al. Glymphatic pathway of gadoliniumbased contrast agents through the brain: overlooked and misinterpreted. Invest Radiol. 2019;54:229-237.

18. Radbruch A. Gadolinium deposition in the brain: we need to differentiate between chelated and dechelated gadolinium. Radiology. 2018;288:434-435.

19. Robert P, Fingerhut S, Factor C, et al. One-year retention of gadolinium in the brain: comparison of gadodiamide and gadoterate meglumine in a rodent model Radiology. 2018;288:424-433.

20. Radbruch A, Roberts DR, Clement O, et al. Chelated or dechelated gadolinium deposition. Lancet Neurol. 2017;16:955.

21. Radbruch A, Richter H, Fingerhut S, et al. Gadolinium deposition in the brain in a large animal model: comparison of linear and macrocyclic gadolinium-based contrast agents. Invest Radiol. 2019;54:531-536.

22. Bower DV, Richter JK, von Tengg-Kobligk H, et al. Gadolinium-based MRI contrast agents induce mitochondrial toxicity and cell death in human neurons, and toxicity increases with reduced kinetic stability of the agent. Invest Radiol. 2019;54:453-463.

23. Radbruch A. How should we measure neurotoxicity of gadolinium-based contrast agents? Invest Radiol. 2019;54:464-465.

24. Semelka RC, Ramalho J, Vakharia A, et al. Gadolinium deposition disease: initial description of a disease that has been around for a while. Magn Reson Imaging. 2016;34:1383-1390.

25. McDonald RJ, Levine D, Weinreb J, et al. Gadolinium retention: a research roadmap from the $2018 \mathrm{NIH} / \mathrm{ACR} / \mathrm{RSNA}$ workshop on gadolinium chelates. Radiology. 2018;289:517-534

26. Semelka RC. Available at: https://www.richardsemelka.com/single-post/ 2019/05/21/Gadolinium-Deposition-Disease-Revised-Symptom-DescriptionMay-2019. 2019.

27. Terkelsen AJ, Karlsson P, Lauria G, et al. The diagnostic challenge of small fibre neuropathy: clinical presentations, evaluations, and causes. Lancet Neurol. 2017; 16:934-944.

28. Lauria G, Hsieh ST, Johansson O, et al, European Federation of Neurological Societies; Peripheral Nerve Society. European Federation of Neurological Societies/ Peripheral Nerve Society Guideline on the use of skin biopsy in the diagnosis of small fiber neuropathy. Report of a joint task force of the European Federation of Neurological Societies and the peripheral nerve society. Eur J Neurol. 2010; 17:903-912, e44-e49.

29. Guidelines A. AVMA Guidelines for the Euthanasia of Animals. 2013. Available at: https://www.avma.org/KB/Policies/Documents/euthanasia.pdf.

30. Lohrke J, Frisk AL, Frenzel T, et al. Histology and gadolinium distribution in the rodent brain after the Administration of Cumulative High Doses of linear and macrocyclic gadolinium-based contrast agents. Invest Radiol. 2017;52:324-333.

31. Pietsch H, Lengsfeld $P$, Jost $G$, et al. Long-term retention of gadolinium in the skin of rodents following the administration of gadolinium-based contrast agents Eur Radiol. 2009;19:1417-1424.

32. Sutresno A, Haryanto F, Viridi S, et al. Diffusion and Interaction bewteen ion $\mathrm{Ca} 2$ + and ion Gd3+ in a Model Synapse: A Monte Carlo Study. Journal of Physics: Conference Series. 2019.

33. Reagan-Shaw S, Nihal M, Ahmad N. Dose translation from animal to human studies revisited. FASEB J. 2008;22:659-661.

34. Blanchard OL, Smoliga JM. Translating dosages from animal models to human clinical trials-revisiting body surface area scaling. FASEB J. 2015;29:1629-1634.

35. Sopacua M, Hoeijmakers JGJ, Merkies ISJ, et al. Small-fiber neuropathy: expanding the clinical pain universe. J Peripher Nerv Syst. 2019;24:19-33.

36. Carozzi VA, Canta A, Chiorazzi A. Chemotherapy-induced peripheral neuropathy: what do we know about mechanisms? Neurosci Lett. 2015;596:90-107.

37. Eldridge S, Guo L, Hamre J 3rd. A comparative review of chemotherapy-induced peripheral neuropathy in in vivo and in vitro models. Toxicol Pathol. 2019; 48:190-201.

38. Ko MH, Chen WP, Hsieh ST. Neuropathology of skin denervation in acrylamideinduced neuropathy. Neurobiol Dis. 2002;11:155-165.

39. Radbruch A, Weberling LD, Kieslich PJ, et al. Response. Radiology. 2016; 279:324-325.

40. Radbruch A. Are some agents less likely to deposit gadolinium in the brain? Magn Reson Imaging. 2016;34:1351-1354.

41. Kleesiek J, Morshuis JN, Isensee F, et al. Can virtual contrast enhancement in brain MRI replace gadolinium?: a feasibility study. Invest Radiol. 2019;54:653-660.

42. Paech D, Schuenke P, Koehler C, et al. T1 $\rho$-weighted dynamic glucose-enhanced MR imaging in the human brain. Radiology. 2017;285:914-922.

43. Schuenke P, Paech D, Koehler C, et al. Fast and quantitative T1 $\rho$-weighted dynamic glucose enhanced MRI. Sci Rep. 2017;7:42093. 